\title{
Efficient anchoring of alien chromosome segments introgressed into bread wheat by new Leymus racemosus genome-based markers
}

Offiong Ukpong Edet ${ }^{1,7}$, June-Sik Kim², Masanori Okamoto ${ }^{3}$, Kousuke Hanada ${ }^{4}$, Tomoyuki Takeda ${ }^{4}$, Masahiro Kishii ${ }^{5}$, Yasir Serag Alnor Gorafi, ${ }^{1,6}$ and Hisashi Tsujimoto ${ }^{1 *}$ (i)

\begin{abstract}
Background: The tertiary gene pool of bread wheat, to which Leymus racemosus belongs, has remained underutilized due to the current limited genomic resources of the species that constitute it. Continuous enrichment of public databases with useful information regarding these species is, therefore, needed to provide insights on their genome structures and aid successful utilization of their genes to develop improved wheat cultivars for effective management of environmental stresses.

Results: We generated de novo DNA and mRNA sequence information of $L$. racemosus and developed 110 polymorphic PCR-based markers from the data, and to complement the PCR markers, DArT-seq genotyping was applied to develop additional 9990 SNP markers. Approximately 52\% of all the markers enabled us to clearly genotype 22 wheat-L. racemosus chromosome introgression lines, and L. racemosus chromosome-specific markers were highly efficient in detailed characterization of the translocation and recombination lines analyzed. A further analysis revealed remarkable transferability of the PCR markers to three other important Triticeae perennial species: L. mollis, Psathyrostachys huashanica and Elymus ciliaris, indicating their suitability for characterizing wheat-alien chromosome introgressions carrying chromosomes of these genomes.

Conclusion: The efficiency of the markers in characterizing wheat-L. racemosus chromosome introgression lines proves their reliability, and their high transferability further broadens their scope of application. This is the first report on sequencing and development of markers from L. racemosus genome and the application of DArT-seq to develop markers from a perennial wild relative of wheat, marking a paradigm shift from the seeming concentration of the technology on cultivated species. Integration of these markers with appropriate cytogenetic methods would accelerate development and characterization of wheat-alien chromosome introgression lines.
\end{abstract}

Keywords: Leymus racemosus (1), Molecular markers (2), DArT-seq (3), Genome sequencing (4), Chromosome introgression lines (5), Salinity stress (6), Triticeae (7)

* Correspondence: tsujim@alrc.tottori-u.ac.jp

${ }^{1}$ Arid Land Research Center, Tottori University, Tottori, Japan

Full list of author information is available at the end of the article 


\section{Background}

Leymus racemosus (mammoth wild rye) and other wild relatives of wheat in the tribe Triticeae (family Poaceae) have, over time, been variously utilized for breeding of hexaploid wheat [1-11]. A typical member of the tribe Triticeeae, L. racemosus is a tetraploid species with 14 linkage groups and seven basic chromosomes in each of its genomes $(2 \mathrm{n}=4 \mathrm{x}=28$; NsNsXmXm) [12]. The genus Psathyrostachys is accepted to be the progenitor source of the Ns genome, but the progenitor of Xm genome has not been ascertained although some recent reports claim that tetraploid Leymus species are segmental polyploids having variant $\mathrm{Ns}$ genomes $\left(\mathrm{Ns}_{1} \mathrm{Ns}_{2}\right)$ of Psathyrostachys $[13,14]$. Leymus racemosus is a renowned sturdy species with high potentials for breeding of bread wheat [15-17].

Mining of useful genes from wild genetic resources, especially the tertiary gene pool, through distant hybridization, to broaden the genetic base of elite cultivars of bread wheat is expected to continue, considering the current trend in global climatic change, accompanied by new strains of pests and disease pathogens. This strategy is, however, largely hindered by linkage drag and low rate of success in distant hybridization, which, in this age of next-generation sequencing (NGS) technologies and improved interspecific hybridization techniques, can effectively be managed. While in vitro culture techniques, example embryo rescue, and induction of homoeologous chromosome recombination have been employed to achieve successful distant hybridization and useful gene recombination, integration of appropriate molecular markers into breeding programs to conduct marker-assisted backcrossing can immensely assist in selecting against deleterious genes, hence fast-tracking the process. Unfortunately, unlike their cultivated counterparts, whose genomes have been extensively analyzed, DNA sequence information and molecular markers of these wild species are limited or completely absent in some cases, culminating in a poor understanding of their genome structures and delay in cultivar development and adequate characterization. This dearth of information, which our research sought to address, largely accounts for the current underutilization of the rich diversity readily available for wheat breeding.

Plant breeders, in various attempts to deal with the aforesaid situation, have had to resort to applying available expressed sequence tags (ESTs) from a few perennial grasses and heterologous markers from annual cereals, example barley, to aid their work [18, 19], but the outcomes, although informative, are hardly satisfactory as a consequence of increased species divergence arising from mutations and other genetic events during speciation. To effectively harness useful genes from these all-important genetic resources, their genomic information base should be continually enriched to at least include data on outstanding species that can serve as representatives for their evolutionary close relatives. Efforts to achieve this much needed expansion have generated enormous molecular cytogenetic data, EST-SSR markers, EST linkage maps and other useful information [12, 19-23]. However, molecular markers developed from whole genome sequence information of these species are still lacking, making it difficult to adequately anchor alien chromosome segments in wheat-alien chromosome introgression lines (CILs). Also, the application of DArT-seq genotyping to study diversity and develop molecular markers from wild Triticeae species is yet to be accorded the popularity it deserves.

In this research, therefore, we applied PCR and DArTseq to develop 8632 [110 PCR-based and 8522 DArT-seq (SNPs)] polymorphic markers from the genome of Leymus racemosus. We also developed additional 1468 CILs-based SNPs which are obvious polymorphisms resulting from the interaction between the alien chromosomes and the background/carrier. Our efforts extended to the application of $5196(\sim 52 \%)$ of all the markers to genotype 22 wheat- $L$. racemosus CILs and the analysis of the transferability of PCR-based markers to other Triticeae species, with emphasis on donor species whose genomes have not been sequenced. This is the first research reporting on the development of molecular markers from $L$. racemosus whole genome and RNA-seq, and the application of DArT-seq platform of wheat to develop DNA markers from its perennial wild relative.

\section{Results}

\section{Development of $L$. racemosus polymorphic markers}

From a total of 294 primer sets screened by PCR, 164 sets ( $56 \%)$ amplified $L$. racemosus genome. Out of the amplified markers, $110(\sim 67 \%)$ were polymorphic in wheat - absence or difference in size of bands in wheat - (Fig. 1a; Table 1). Six of the polymorphic markers showed size polymorphism, while 104 markers constituted presence/absence polymorphism. Also, out of 11,570 DArT-seq SNP markers filtered based on high call and reproducibility rates, 8522 $(\sim 74 \%)$ were polymorphic in wheat (absence of SNP alleles in wheat) -8430 SNPs were absent in our wheat cultivar, CS, while 92 were present but showed presence of both reference and SNP alleles in L. racemosus (Fig. 1c; Table 1). These 92 markers form part of the polymorphisms we observed between our CS and the reference CS genome sequence on DArT platform. Taken together, we developed a total of 8632 polymorphic markers from L. racemosus genome.

\section{Characterization of wheat- $L$.racemosus chromosome introgression lines with $L$. racemosus markers}

About 65\% (72 markers) of the polymorphic PCR markers amplified $L$. racemosus chromosomes in nine wheat- $L$. racemosus chromosome addition lines, while 


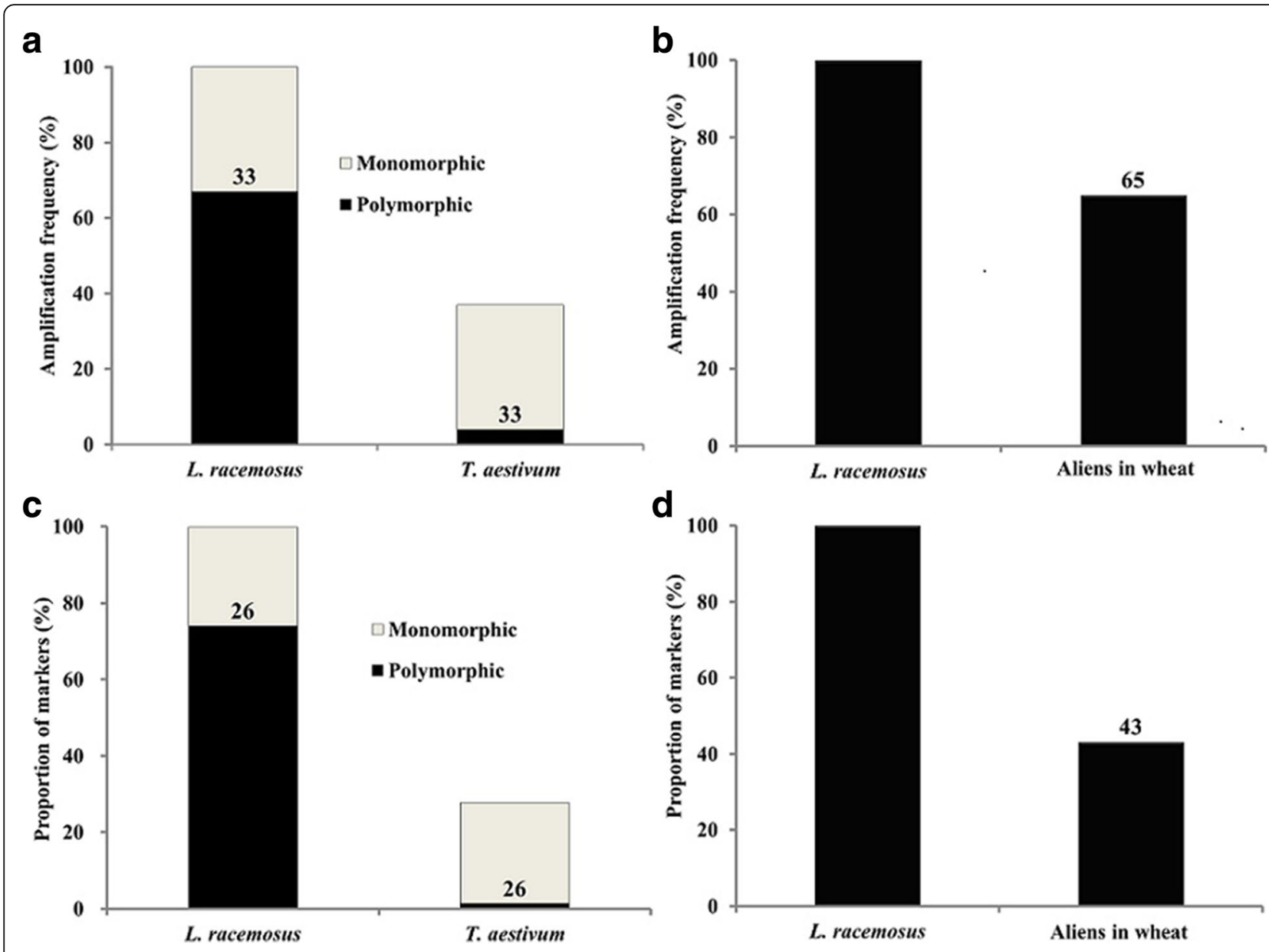

Fig. 1 Analysis of markers located on L. racemosus, bread wheat and nine wheat-L. racemosus chromosome addition lines. a Amplification of 164 pre-screened L. racemosus PCR-based markers in L. racemosus and bread wheat genomes. b Amplification of 110 polymorphic L. racemosus PCRbased markers in nine wheat-L. racemosus addition lines, with $L$. racemosus genome as a positive control. c Differentiation of $L$. racemosus and bread wheat genomes using 11,570 SNPS. d Total SNPs (8522) located in nine wheat-L. racemosus addition lines

Table 1 Identification of wheat-L. racemosus chromosome introgression lines using polymorphic markers from L. racemosus genome

\begin{tabular}{|c|c|c|c|c|c|c|c|}
\hline \multirow[t]{2}{*}{ Genotype ID } & \multirow[t]{2}{*}{ Description } & \multirow{2}{*}{$\begin{array}{l}\text { Chromosome } \\
\text { constitution } \\
\text { (2n) }\end{array}$} & \multicolumn{2}{|c|}{ Total number of markers } & \multicolumn{3}{|c|}{ Chromosome-specific markers } \\
\hline & & & $P C R$ & DArT-seq & PCR & DArTseq & Total \\
\hline TACBOW 0001 & Disomic addition & $21 "+1 "[L r \# A]$ & 14 & 434 & 6 & 381 & 387 \\
\hline TACBOW 0003 & Disomic addition & $21 "+1 "[\mathrm{Lr} \# \mathrm{E}]$ & 4 & 46 & 1 & 1 & 2 \\
\hline TACBOW 0004 & Disomic addition & $21 "+1 "[\mathrm{Lr} \# \mathrm{~F}]$ & 10 & 499 & 4 & 344 & 348 \\
\hline TACBOW 0005 & Disomic addition & $21 "+1 "[\mathrm{Lr} \# \mathrm{H}]$ & 20 & 528 & 12 & 410 & 422 \\
\hline TACBOW 0006 & Disomic addition & $21 "+1 "[$ Lr\#l] & 12 & 558 & 4 & 491 & 495 \\
\hline TACBOW 0007 & Disomic addition & $21 "+1 "[$ Lr\#J $]$ & 17 & 511 & 9 & 450 & 459 \\
\hline TACBOW 0008 & Disomic addition & $21 "+1 "[\mathrm{Lr} \# \mathrm{~K}]$ & 12 & 519 & 7 & 465 & 472 \\
\hline TACBOW 0009 & Disomic addition & $21 "+1 "[L r \# L]$ & 18 & 647 & 8 & 525 & 533 \\
\hline TACBOW 0010 & Disomic addition & $21 "+1 "[$ Lr\#N] & 16 & 549 & 5 & 428 & 433 \\
\hline All aliens & - & - & 72 & 3656 & 56 & 3495 & 3551 \\
\hline KT020-003 (CS) & Triticum aestivum & 42 & 6 & 92 & - & - & - \\
\hline TACBOW 0112 & Leymus racemosus & 28 & 110 & 8522 & - & - & - \\
\hline
\end{tabular}

TACBOW Tottori Alien Chromosome Bank of Wheat (Japan), Lr Leymus racemosus, $A, E, F, H, I, J, K, L, N$ Arbitrary numbering of $L$. racemosus chromosomes;": bivalent; Bold numbers: all markers located on alien chromosomes in wheat background in each category; CS: Chinese Spring 
approximately 43\% (3656 SNPs) of the DArT-seq (SNP) markers identified the nine alien chromosomes (Table 1; Fig. $1 \mathrm{~b}$ and $\mathrm{d}$ ). It should be noted here that we used only SNP markers from the DArT-seq data in our analysis, as silico DArT was less informative in analyzing the required polymorphism. This is because silico DArT data is binary (dominant), making it impossible to identify polymorphism as codominance (in our case, presence of both reference and SNP alleles), which we mostly utilized to genotype the chromosome introgression lines, since they have genome representations of wheat (alien chromosome recipient) and L. racemosus (alien chromosome donor).

\section{Development of L. racemosus chromosome-specific markers}

We developed a total of 3551 chromosome-specific markers for the nine $L$. racemosus chromosomes in wheat genetic background, and the number of the specific markers per chromosome ranged between two in Lr\#E and 533 in Lr\#L (Table 1; Additional files 1, 2, 3, 4, 5, 6, 7, 8 and 9). The large number of markers on each chromosome enabled us to reliably differentiate the nine wheat- $L$. racemosus chromosome addition lines analyzed (Table 1).

\section{Confirmation of homoeologous groups of L. racemosus chromosomes in wheat background}

To further assess the validity of our chromosome-specific SNP markers, we exploited correspondence of L. racemosus chromosome-specific markers with the homoeologous groups of CS chromosomes to determine the most probable homoeologous group (HG) of each L. racemosus chromosome in the chromosome addition lines (Table 2). The results revealed that the alien chromosomes spread between HG 2 and 7: Lr\#A and L are in HG 2, Lr\#H and N are in HG 3, while Lr\#F, I, K and J are in HG 4, 5, 6 and 7, respectively.
Detailed characterization of chromosomes I, J, N and their respective translocation arms

As shown in Table 3, we successfully allocated I-, J- and $\mathrm{N}$-specific markers to their respective arms using their respective translocation lines.

A detailed analysis of the homology between each of the three chromosomes and their respective translocated arms gave a clearer picture of the structures of the translocation lines. For chromosome I, the markers were adequately allocated to the short (S) and long (L) arm translocations, revealing the proportions of chromosome I markers that differentiated each of the translocated arms and eight markers located on a segment of chromosome I that may have not been transmitted during the production of the translocation lines (Fig. 2a). However, we observed few markers specific to the translocation lines, which are absent in I-addition line. If no genotyping error is assumed, these markers would represent polymorphisms that may have arisen from the interactions between the translocated arms and CS genome.

Most J-chromosome markers were found to be present only in the JS translocation, about half of which were co-located on the JL translocation (Fig. 2b). This result obviously indicates that what we hitherto regarded as JL translocation is a segment of JS translocation. The 10 unique markers (Fig. 2b) each present in the two translocation lines may have resulted from changes in each genetic background or small chromosomal rearrangements during the various production processes. As observed in chromosome I, 14 markers identified a segment of chromosome J which may have not been transmitted to the translocation lines.

Chromosome $\mathrm{N}$ and its translocated arms presented a scenario similar to chromosome I. Both the NS and NL arm translocations of chromosome $\mathrm{N}$ showed well separated markers (Fig. 2c). However, 27 markers were found

Table 2 Determination of homoeologous groups of L. racemosus chromosomes in wheat genetic background using chromosomespecific DArT-seq SNP markers

\begin{tabular}{|c|c|c|c|c|c|c|c|c|c|c|c|c|c|c|c|c|c|c|c|c|c|c|c|}
\hline \multirow[t]{2}{*}{ Alien } & \multicolumn{21}{|c|}{$\begin{array}{l}\text { Number of markers corresponding to each homoeologous group of bread wheat (Chinese Spring) } \\
\text { chromosome }\end{array}$} & \multicolumn{2}{|c|}{$\begin{array}{l}\text { Most probable HG of alien } \\
\text { chromosome }\end{array}$} \\
\hline & $\overline{1 A}$ & $1 \mathrm{~B}$ & $1 D$ & $2 \mathrm{~A}$ & $2 B$ & $2 \mathrm{D}$ & $3 A$ & $3 B$ & $3 D$ & $4 \mathrm{~A}$ & $4 \mathrm{~B}$ & $4 D$ & $5 \mathrm{~A}$ & $5 B$ & $5 D$ & $6 \mathrm{~A}$ & $6 B$ & $6 D$ & $7 \mathrm{~A}$ & $7 B$ & $7 \mathrm{D}$ & Previous reports & Current \\
\hline$\overline{L r \# A}$ & 3 & 1 & 1 & 75 & 71 & 198 & 3 & 0 & 4 & 0 & 2 & 2 & 1 & 0 & 5 & 1 & 3 & 5 & 2 & 3 & 1 & $2^{a, b}$ & 2 \\
\hline Lr\#E & 0 & 0 & 0 & 0 & 0 & 0 & 0 & 0 & 0 & 0 & 0 & 0 & 0 & 0 & 1 & 0 & 0 & 0 & 0 & 0 & 0 & $N D$ & $N D$ \\
\hline Lr\#F & 4 & 2 & 3 & 1 & 2 & 5 & 0 & 0 & 0 & 77 & 79 & 141 & 2 & 12 & 20 & 0 & 2 & 0 & 3 & 1 & 2 & $4^{a, b}$ & 4 \\
\hline Lr\#H & 1 & 2 & 7 & 0 & 2 & 2 & 68 & 94 & 204 & 2 & 2 & 4 & 3 & 1 & 2 & 0 & 6 & 2 & 0 & 4 & 4 & $3^{a, b}$ & 3 \\
\hline Lr\#I & 3 & 0 & 5 & 3 & 1 & 4 & 1 & 1 & 5 & 2 & 7 & 9 & 74 & 162 & 193 & 4 & 2 & 4 & 3 & 6 & 2 & $5^{a, b}$ & 5 \\
\hline Lr\#」 & 1 & 0 & 3 & 4 & 2 & 5 & 2 & 2 & 4 & 12 & 2 & 4 & 5 & 7 & 2 & 0 & 3 & 5 & 84 & 105 & 208 & $(6,7)^{a},(3,7)^{b}$ & 7 \\
\hline Lr\#K & 4 & 2 & 4 & 1 & 3 & 7 & 1 & 2 & 2 & 0 & 2 & 6 & 2 & 2 & 6 & 81 & 117 & 205 & 5 & 8 & 9 & $6^{a, b}$ & 6 \\
\hline Lr\#L & 1 & 0 & 1 & 135 & 158 & 293 & 1 & 6 & 6 & 3 & 3 & 2 & 2 & 7 & 9 & 0 & 3 & 6 & 11 & 5 & 5 & $2^{a, b}$ & 2 \\
\hline Lr\#N & 4 & 6 & 3 & 0 & 1 & 4 & 77 & 131 & 176 & 3 & 0 & 0 & 1 & 3 & 3 & 0 & 2 & 1 & 2 & 4 & 5 & $2^{a},(3,7)^{b}$ & 3 \\
\hline
\end{tabular}

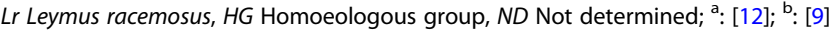

The bold numbers represent the number of markers indicating the homoeologous groups of $L$. racemosus chromosomes 
Table 3 Determination of arm-specific markers of chromosomes Lr\#l, Lr\#J and Lr\#N based on markers specific to their translocated arms

\begin{tabular}{|c|c|c|c|c|c|}
\hline \multirow{2}{*}{$\begin{array}{l}\text { Genotype } \\
\text { ID }\end{array}$} & \multirow[t]{2}{*}{ Description } & \multirow{2}{*}{$\begin{array}{l}\text { Chromosome } \\
\text { constitution } \\
(2 n)\end{array}$} & \multicolumn{3}{|c|}{ Arm-specific markers } \\
\hline & & & PCR & DArTseq & Total \\
\hline I short & I short arm translocation & 42 & 1 & 80 & 81 \\
\hline I long & I long arm translocation & 42 & 0 & 404 & 404 \\
\hline J short & J short arm translocation & 42 & 5 & 231 & 236 \\
\hline J long & $\mathrm{J}$ long arm translocation & 42 & 0 & 2 & 2 \\
\hline$N$ short & $\mathrm{N}$ short arm translocation & 42 & 2 & 162 & 164 \\
\hline N long & $\mathrm{N}$ long arm translocation & 42 & 3 & 255 & 258 \\
\hline
\end{tabular}

to be specific to NL and nine specific to NS translocation lines (Fig. 2c), indicating unique polymorphisms which may have been acquired from interactions between the background and the translocated arms as observed for chromosomes I and J. Also, the 13 markers located on the whole $\mathrm{N}$-addition line, which are absent in the translocated arms, suggest that the NS and NL translocations lost the region of chromosome $\mathrm{N}$ identified by these markers (Fig. 2c).

\section{Analysis of recombination positions of $\mathrm{N}$-recombination lines}

The N-specific markers aided us to determine the size of the recombinant fragments and map their locations on Lr\#N and the corresponding CS chromosomes, revealing the probable fraction of CS chromosome replaced in each recombination line (Table 4; Fig. 3). N recombinant fragments 2, 3, 5 and 6 were found to be located in the short arm, while the recombinant fragments 4 and 7 were found in the long arm of each of the lines. Although the two markers that specified recombinant fragment 1 were traced to NL, we are not certain about the arm location of this fragment; hence, we intend to clarify this in a future report. Recombination lines 6 and 7 were observed to have the largest fragments, with all the markers in the short arm translocation represented in recombination line 6 , and all except two markers in long arm translocation represented in recombination line 7 (Table 4; Fig. 3). Other lines were found to have relatively small fragments which can best be described as different sizes of bins represented in recombination lines 6 or 7 . With the two markers recorded for recombination line 1 (Table 4), it would appear as though there was no recombination event, although low recombination rates between wheat chromosomes and aliens is not unusual [24]. However, molecular cytogenetic characterization clearly differentiated the lines (Additional file 10), indicating the importance of integrated characterization of wheat-alien CILs.

\section{Leymus racemosus Chromosomes' universal markers}

Two of the polymorphic markers, 21_s46518 and 333_s46518 (developed from the same DNA sequence scaffold) identified all the $L$. racemosus chromosomes in wheat (Fig. 4a; Additional file 11). On sequencing PCR products generated with one of these markers, and conducting BLAST search with the official NCBI search tool (BLASTN, megablast), we observed that $26 \%$ and $16 \%$ of sequences of
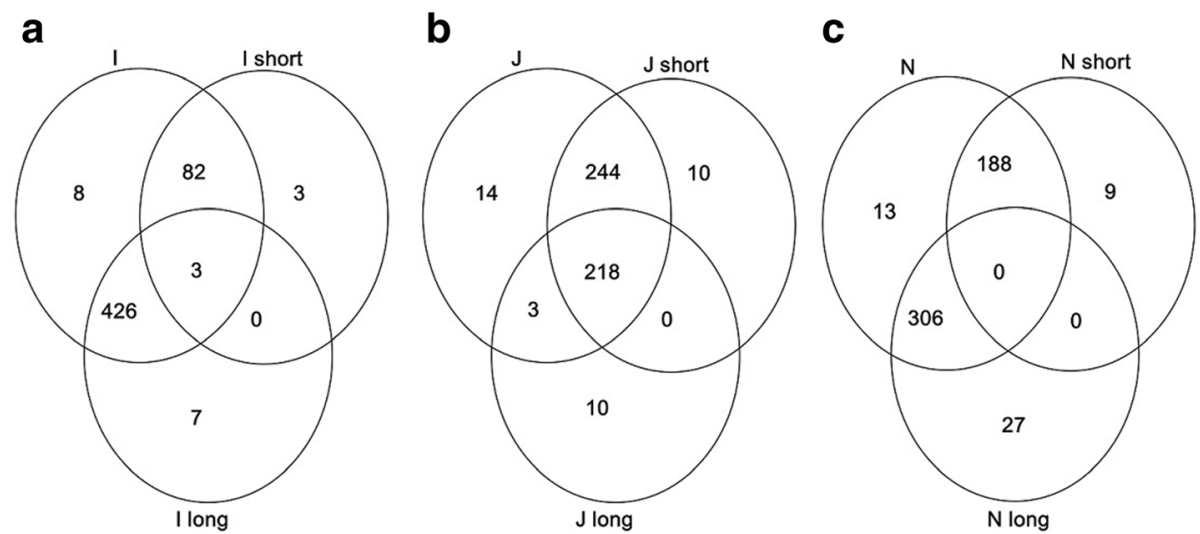

Fig. 2 Venn diagrams showing homology and polymorphism between chromosomes I, J, N and their respective translocation lines. a homology and polymorphism between I and its translocated arms. $\mathbf{b}$ homology and polymorphism between $\mathrm{J}$ and its translocated arms. $\mathbf{c}$ homology and polymorphism between $\mathrm{N}$ and its translocated arms 
Table 4 Determination of arm locations of N-recombinant fragments using arm-specific markers

\begin{tabular}{|c|c|c|c|c|c|}
\hline \multirow[t]{3}{*}{ Genotype } & \multicolumn{4}{|c|}{ Arm location of amplified markers } & \multirow{3}{*}{$\begin{array}{l}\text { Fragment } \\
\text { location }\end{array}$} \\
\hline & \multicolumn{2}{|l|}{ PCR } & \multicolumn{2}{|c|}{ DArTseq } & \\
\hline & Short & Long & Short & Long & \\
\hline N recombination No. 1 & 0 & 0 & 0 & 2 & Not certain \\
\hline N recombination No. 2 & 2 & 0 & 160 & 0 & Short arm \\
\hline N recombination No. 3 & 0 & 0 & 67 & 0 & Short arm \\
\hline N recombination No. 4 & 0 & 1 & 0 & 48 & Long arm \\
\hline N recombination No. 5 & 2 & 0 & 158 & 0 & Short arm \\
\hline N recombination No. 6 & 2 & 0 & 162 & 0 & Short arm \\
\hline N recombination No. 7 & 0 & 3 & 0 & 248 & Long arm \\
\hline $\mathrm{N}$ short arm translocation & 2 & - & 162 & - & - \\
\hline $\mathrm{N}$ long arm translocation & - & 3 & - & 255 & - \\
\hline
\end{tabular}

the PCR products of $L$. racemosus and wheat-Lr\#N, respectively aligned to a section of CACTA-family transposon in Lolium perenne (perennial rye-grass), a highly researched commercial pasture crop of the grass family, Poaceae. Sequences of other CILs showed no significant alignment to the transposon sequence.

\section{Additional unique CILs-based SNPs}

DArT-seq data further revealed additional 1468 unique SNPs in the nine wheat $L$. racemosus addition lines, absent in the two parents. One hundred and ninetyseven of these SNPs are common to the lines, while 1271 are line-specific, with a range of 38-355 specific markers on each line (Table 5). Like the L. racemosus chromosome-specific markers, the line-specific markers also guided us in differentiating the nine addition lines. These additional SNPs account for polymorphisms acquired from the interactions between the added chromosomes and the background (CS genome), and their effects may be of agronomic significance.

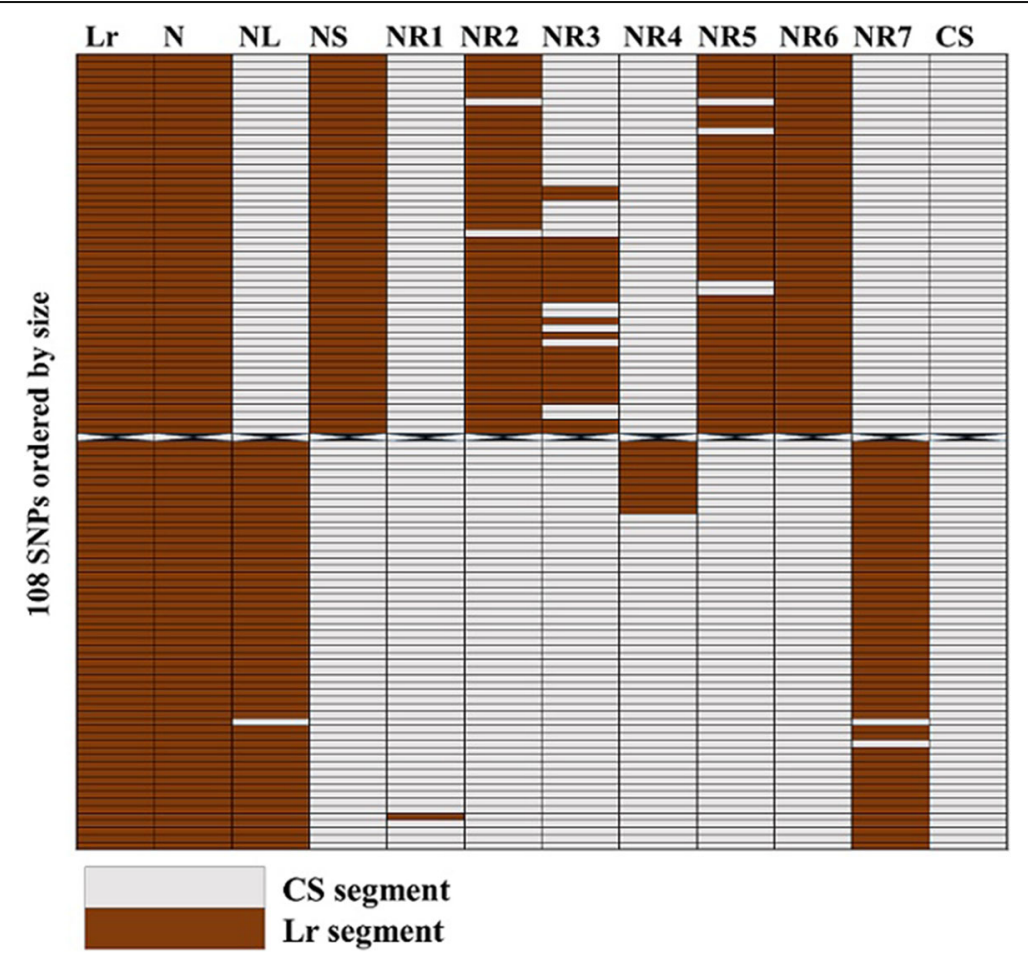

Fig. 3 Graphical genotyping of N-recombination lines using 108 chromosome N-specific SNPs corresponding to wheat chromosome 3B. Lr: Leymus racemosus, N: N-addition line, NL: N long arm translocation line, NS: N short arm translocation line, NR1-NR7: N recombination lines 1-7, CS: bread wheat cv. Chinese Spring 


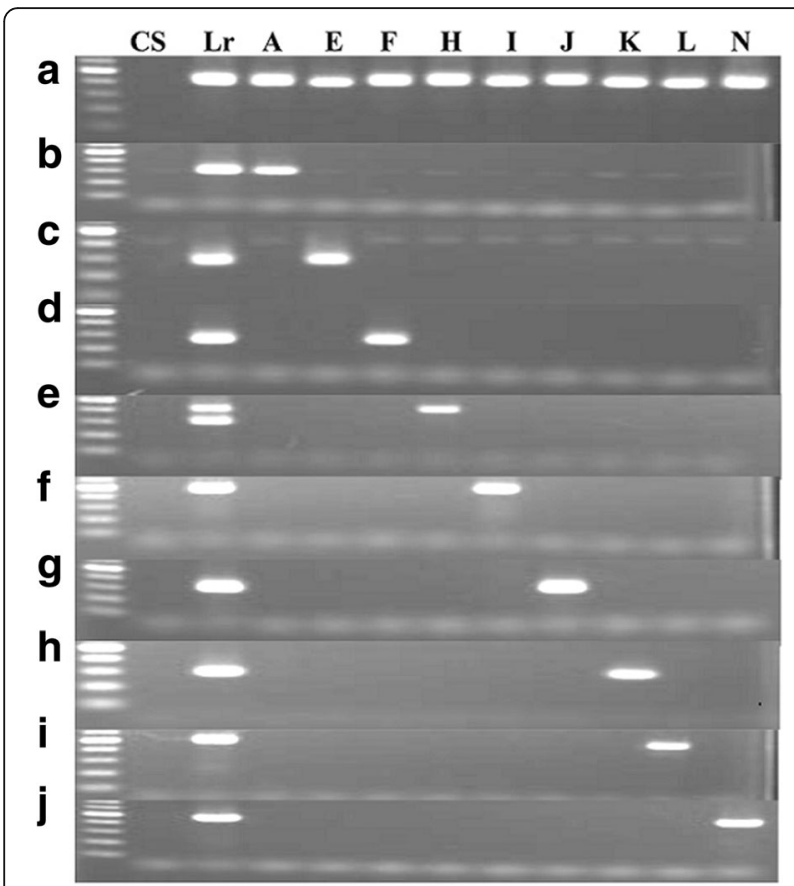

Fig. 4 Representative gels of PCR amplification of wheat, $L$. racemosus and wheat- $L$. racemosus chromosome addition lines. a Amplification of nine $L$. racemosus chromosomes in wheat background by a universal marker. $\mathbf{b}-\mathbf{j}$ Amplification of the nine chromosomes by their respective PCR-based chromosome-specific markers. CS: Chinese Spring; Lr: Leymus racemosus; A, E, F, H, I, J, L, N: Nine wheat-Leymus racemosus addition lines

\section{Analysis of transferability of $L$. racemosus markers to other Triticeae species}

To assess the applicability of $L$. racemosus markers in studying the genomes of other related species, we analyzed the transferability of the markers using genomic
DNA from 11 other Triticeae species, alongside with $L$. racemosus. The results of this analysis, utilizing 164 prescreened $L$. racemosus PCR-based markers, showed that $75 \%$ of the markers were transferable, while the remaining $25 \%$ were $L$. racemosus genome-specific, particularly revealing higher amplification frequencies in three other important perennial Triticeae species ( $L$. mollis, Psathyrostachys huashanica and Elymus ciliaris) in comparison to wheat and other species studied (5a-d; Table 6). More importantly, the amplified markers in each of these species were found to be reasonably polymorphic in wheat (Table 6), obviously indicating their suitability in genotyping wheat-alien CILs carrying chromosomes from these species.

Interestingly, the two universal markers which identified all $L$. racemosus chromosomes in wheat genetic background were found to be Leymus-specific, as they amplified only the two Leymus species out of the 12 Triticeae species analyzed, revealing size polymorphism between the two Leymus genomes (Fig. 5b). These markers can, therefore, be applied to separate Leymus genomes from genomes of other species in the same tribe, and their (Leymus) chromosomes, if introgressed into wheat, can easily be sorted out in one PCR. We also observed informative co-amplification between the two Leymus species and Psathyrostachys huashanica (Fig. 5c), and a phylogenetic analysis using 123 markers co-amplified among the 12 Triticeae species (Fig. 6) revealed a close evolutionary relationship between the three species, which agrees with reports asserting that Leymus species are segmental polyploids with variant $\mathrm{N}$-genomes from genus Psathyrostachys [13, 25]. However, one highly conserved marker sequence amplified all the species, revealing size polymorphism among them (Fig. 5d).

Table 5 Additional 1468 unique genotype-based SNPs located on nine wheat-Leymus racemosus chromosome addition lines

\begin{tabular}{llll}
\hline Genotype ID & Alien chromosome ID & Common SNPs on each chromosome & Line-specific SNPs \\
\hline TACBOW 0001 & Lr\#A & 99 & 355 \\
TACBOW 0003 & Lr\#E & 79 & 38 \\
TACBOW 0004 & Lr\#F & 70 & 173 \\
TACBOW 0005 & Lr\#H & 86 & 109 \\
TACBOW 0006 & Lr\#\# & 74 & 46 \\
TACBOW 0007 & Lr\#J & 68 & 130 \\
TACBOW 0008 & Lr\#K & 61 & 186 \\
TACBOW 0009 & Lr\#L & 92 & 124 \\
TACBOW 0010 & Lr\#N & 81 & 110 \\
KT020-003 (CS) & - & 0 & 0 \\
TACBOW 0112 (Lr) & - & 0 & $\mathbf{1 2 7 1}$ \\
Total & & $\mathbf{1 9 7}$ & 0 \\
\hline
\end{tabular}

TACBOW Tottori Alien Chromosome Bank of Wheat (Japan), CS Chinese Spring, Lr Leymus racemosus;197 represents the total number of SNPs common to the nine CILs, without repetition 
Table 6 Transferability of 164 pre-screened L. racemosus markers to 11 other Triticeae species

\begin{tabular}{|c|c|c|c|c|c|c|}
\hline \multirow[t]{2}{*}{ Species } & \multicolumn{3}{|c|}{ Amplified markers } & \multirow{2}{*}{$\begin{array}{l}\text { Polymorphism } \\
\text { to wheat (\%) }\end{array}$} & \multicolumn{2}{|c|}{ Polymorphism based on marker source (\%) } \\
\hline & DNA & RNA & Total & & DNA & RNA \\
\hline Leymus racemosus & 76 & 88 & $164(100)$ & 67 & 83 & 53 \\
\hline L. mollis & 31 & 64 & $95(58)$ & 49 & 74 & 38 \\
\hline Psathyrostachys huashanica & 23 & 55 & $78(\mathbf{4 8})$ & 44 & 70 & 33 \\
\hline Elymus ciliaris & 17 & 49 & $66(40)$ & 32 & 42 & 29 \\
\hline Hordeum vulgare & 5 & 31 & $36(22)$ & 22 & 0 & 16 \\
\hline H. bulbosum & 8 & 31 & 39 (24) & 23 & 25 & 23 \\
\hline Dasypyrum villosum & 9 & 39 & $48(29)$ & 21 & 33 & 18 \\
\hline Secale cereale & 9 & 38 & $47(29)$ & 15 & 11 & 16 \\
\hline Triticum urartu & 9 & 36 & $45(27)$ & 7 & 0 & 8 \\
\hline Aegilops speltoides & 8 & 39 & $47(29)$ & 6 & 13 & 5 \\
\hline Ae. Tauschii & 10 & 39 & $49(30)$ & 0 & 0 & 0 \\
\hline T. aestivum & 10 & 51 & $61(37)$ & - & - & - \\
\hline L. racemosus-specific markers & 33 & 8 & $41(25)$ & - & - & - \\
\hline
\end{tabular}

Bold numbers in brackets represent percentages of amplified markers; L. racemosus-specific markers represent the proportion of the 164 markers not transferable to the other Triticeae species analyzed

\section{Analysis of polymorphism based on DNA and RNA markers}

In a bid to compare the performance of markers developed from DNA and RNA-seq, we analyzed polymorphism based on the two sources of markers. As expected, markers from genomic sequence were more polymorphic than those from RNA-seq, indicating that the polymorphisms between hexaploid wheat and other Triticeae species studied are more traceable to the

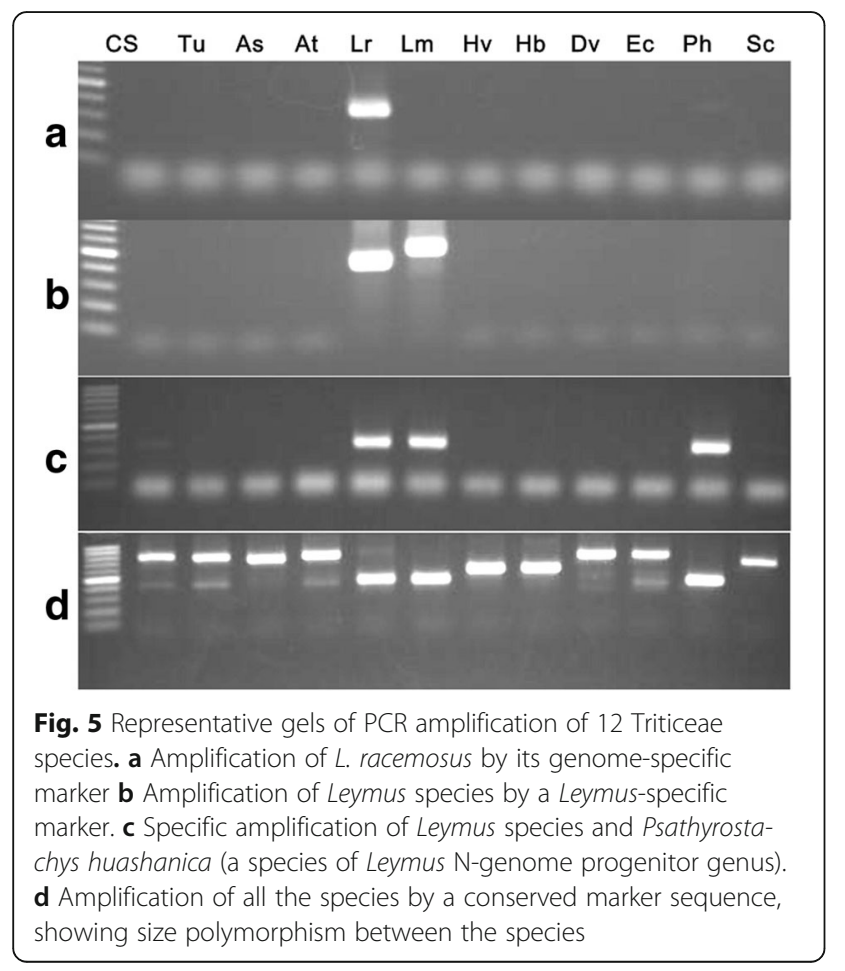

variations in the repetitive sequences of the genomes (Table 6). However, the polymorphisms recorded from the RNA-seq/gene markers (Table 6), which account for variations in the genic regions, make the two approaches equally informative.

\section{Discussion}

Fast-tracking introgression breeding and wheat-alien characterization with appropriate molecular markers

Utilizing introgressive hybridization to combat the agelong wheat genetic erosion has since been identified and is currently inevitable, but the achievements are still not satisfactory mostly resulting from poor understanding of the genomics of important wild relatives of wheat [3, 26].Therefore, to create the necessary platform for successful breeding of hexaploid wheat through the intermediary of its tertiary gene pool, mobilization of research resources towards genome analysis and development of molecular markers from notable Triticeae perennial species must be intensified. The projected $60 \%$ increase in wheat demand in 2050 [27], which obviously cannot be met solely through the cultivation of high yielding elite wheat cultivars, most of which are poorly adapted to harsh growing conditions, further justifies our opinion.

From the stand point of our results (Tables 1-4; Fig. 3), it is evident that the availability of adequate molecular markers from the genomes of potential gene donors can accelerate introgression breeding, as they can be reliably deployed to genotype wheat-alien CILs and tackle linkage drag, where necessary. The massive chromosome-specific markers developed for each of the chromosome addition lines (except Lr\#E) are, therefore, expected to aid breeders 


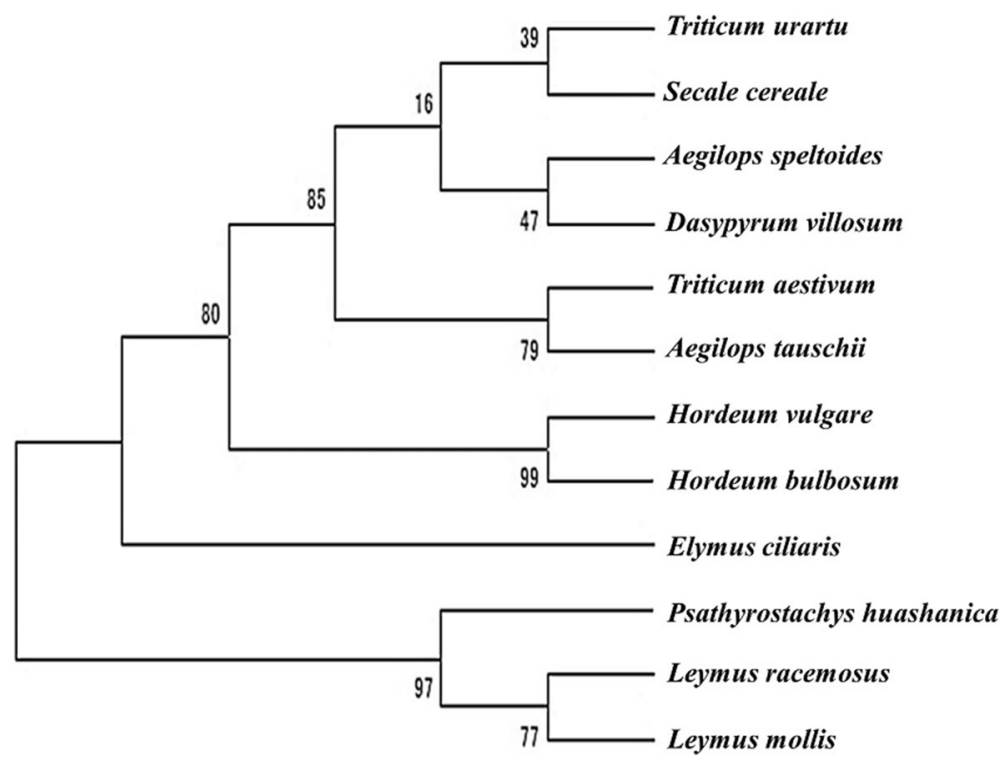

Fig. 6 Phylogenetic tree constructed from the co-amplification of 123 PCR-based Leymus racemosus markers using UPGMA as clustering method

in conducting more stringent screening and selection in their efforts to develop cultivars with only necessary chromosome segments to satisfy specific breeding goals within a reasonable time frame. At the moment, we attribute the few chromosome-specific markers developed for Lr\#E to high homology between wheat genome and the chromosome, having observed about 30\% monomorphic markers between $L$. racemosus and $T$. aestivum genomes (Fig. 1a and c). However, there may be some form of genetic instability, cytochimerism for instance, which we intend to ascertain in the future. We are certain that alien chromosome loss is not the reason for the strange result, as we used the original stocks of all the addition lines in the TACBOW gene bank, which had been characterized by some of our co-authors $[9,28]$, to confirm our results.

The difference between the total number of markers developed and the number of markers that identified the aliens in wheat background (Table 1; Fig. 1b and d) account for the difference between the whole set of chromosomes in $L$. racemosus genome and the number of $L$. racemosus chromosomes we studied in the nine genotypes. Another factor likely to contribute to this difference is the possibility of losing some segments of the nine chromosomes during production of the lines.

Noteworthy is that the higher proportion of PCR markers that identified aliens in wheat as compared to SNP markers (Fig. 1b and d) was expected because SNP markers are sequence-based, which are theoretically more stringent than PCR-based markers. However, the proportions of both the PCR-based and SNP markers that amplified the nine chromosome addition lines are deemed reasonably high, given that $L$. racemosus has 14 pairs of chromosomes, 6 of which are obviously not represented in the nine genotypes analyzed. Also, the effectiveness of the chromosome-specific SNP markers in determining the homoeologous groups of L. racemosus chromosomes further validates the importance of inclusion of appropriate molecular markers in wheat-alien cultivar development and screening. The homoeologous groups of the chromosomes determined by our analysis are highly consistent with previous reports $[9,12]$. Interestingly, we could clarify the homoeologous groups of chromosomes Lr\#J (HG 7) and Lr\#N (HG 3), which were previously not reported with certainty (Table 2). We presume that the chromosomes found to be in the same groups [Lr\#A and Lr\#L (HG 2); Lr\#H and Lr\#N (HG 3)] are homoeologous chromosomes from the two genomes that constitute $L$. racemosus. Our inability to determine the HG of Lr\#E chromosome, as was the case with earlier reports cited here, is another pointer that the line may be genetically unstable.

In our opinion, this approach of utilizing chromosome-specific genome-based molecular markers to characterize introgression lines is faster than in situ hybridization procedures (FISH and GISH), which are traditionally employed for this purpose. Although in situ hybridization methods have proven to be reliable in characterizing CILs, they are lengthy, laborious and not without limitations. An example of such limitations was reported when two genomic in situ hybridization (GISH) procedures failed to reveal some distally located breakpoints in wheat-rye recombinant genotypes [29]. Beyond being easier, characterization of wheat-alien CILs by molecular markers, as proven by our results, brought to light detailed chromosome segments rearrangements, some of which can be likened to "zebra" chromosome 
[30, 31]. Additionally, the unique line-specific polymorphisms revealed by DArT-seq analysis, absent in either of the parents, would not be captured by in situ hybridization methods, as hybridization probes are usually designed to track alien segments, not polymorphisms which may arise from genome interactions. Nevertheless, we are not suggesting the replacement of hybridization procedures with molecular markers. Rather, our strong recommendation is the integration of efficient DNA markers with in situ hybridization strategies in wheat-alien breeding programs to accelerate the process and improve outcomes.

\section{Possibility of genome or alien modification in wheat-alien translocations}

The interactions between alien chromosomes and carrier genomes need to be properly dissected. Analyses of the chromosome addition and translocation lines in our study indicated the possibility of genetic modification of either the introgressed chromosomes, background (wheat genome) or both. These modifications, capable of generating additional polymorphisms, as observed in our study (Table 5; Fig. 2), may result from small chromosomal rearrangements, activation of transposable elements or any other interactive genetic event between alien materials with the genome of wheat [31, 32]. Also, by graphically genotyping the recombination lines, we were able to uncover different patterns of recombination events in each line (Fig. 3). This observation indicates that the same chromosome $(\mathrm{Lr} \# \mathrm{~N})$ interacted with wheat genome in different ways to produce different genotypes, which are likely to result in diversity in agronomic traits. Of more importance is the potential effect of these interactions on the overall performance of the genotypes [33], necessitating detailed studies to clarify the underlying mechanism of such genetic events and their agronomic implications. Such studies would be greatly enhanced by the availability of adequate molecular markers to track aliens and unique polymorphisms which may result from genome interactions.

\section{Association of $L$. racemosus chromosomes' universal markers with CACTA-family transposons}

The universal markers we developed are particularly valuable since they can be applied to easily track the transmission of alien chromosomes over generations, given the possibility of alien chromosome elimination in the course of cultivar multiplication and maintenance [34-36]. Following the alignment of the sequence of one of these markers to CACTA-family transposon in $L$. perenne, we speculate that this marker sequence is part of a possible CACTA-family transposon in Leymus. CACTAfamily transposons, one of the most abundant superfamilies of class II transposons exclusively found in plants, have been reported to play significant roles in genome variation in Triticeae and other plants [37-41]. Although the specific role of this sequence in Leymus species is unknown at the moment, it is likely to have amplified after differentiation of the ancestral species of Leymus.

\section{Leymus chromosome $\mathrm{N}$-specific markers and biological ni- trification inhibition (BNI) activity}

Biological nitrification inhibition (BNI) activity in $L$. racemosus, a highly desirable trait with agronomic and environmental consequences, had previously been reported to be chiefly controlled by chromosome $\mathrm{N}$ [42] The $\mathrm{N}$-specific markers are, therefore, particularly of high value, as they can easily be applied to identify genotypes with BNI activity, avoiding the cumbersome and expensive process of root exudates analysis [43], requiring expertise which an ordinary plant breeder may not possess. Interestingly, only DNA sequences of the PCR products of $L$. racemosus and wheat-Lr\#N generated with one of our universal markers aligned to the CACTA-family transposon in $L$. perenne, one of the forage grasses reported to have endogenous BNI activity [42, 43]. However, whether BNI activity is linked with actions of mobile genetic elements, transposons in this case, cannot be ascertained at the moment.

\section{Transferability of markers between $L$. racemosus and other Triticeae perennials}

Sequencing of all the potential gene sources for wheat breeding in the near future is not expected. Hence, transferability of markers between useful species of this gene pool, as a compensational approach of analysis, is highly desired [44-47]. Our analysis has proven clearly that markers from $L$. racemosus can be successfully transferred to L. mollis, P. huashanica and E. ciliaris, three other important species in the tribe Triticeae (Table 6). Also, the transferred markers were found to be reasonably polymorphic in wheat (Table 6), suggesting their suitability for characterizing wheat genotypes with alien chromosomes from the three genomes. The genera of these species, because of their recognition as profitable forage grasses and gene mines for hexaploid breeding, have received fair research attention $[13,14$, $23,25,48-50]$. However, their genomes have not yet been sequenced, leaving breeders with the option of transferring markers from evolutionary closely related species to analyze their genomes and wheat genotypes carrying their chromosomes.

\section{Conclusion}

The molecular markers developed in this study are expected to play valuable roles in hexaploid wheat breeding, particularly in the process of developing and characterizing wheat-alien CILs. Our success in applying 
them to unequivocally genotype 22 wheat- $L$ racemosus CILs validates their usefulness. Specifically, the universal and $\mathrm{N}$-specific markers are of great breeding importance. While the universal markers can readily be applied to monitor and confirm alien presence and transmission, $\mathrm{N}$-specific markers can find application in mapping of nucleotide sequences associated with biological nitrification inhibition (BNI) activity. The additional SNPs found on the nine chromosome addition lines would be especially useful in identifying and analyzing unique polymorphisms which may result from alien interaction with background, while the $L$. racemosus markers not mapped on any of the nine chromosomes reported here would aid production of other wheat- $L$. racemosus CILs carrying other chromosomes of $L$. racemosus. Also, the remarkable transferability of the PCR-based markers to three other notable perennial Triticeae species is an added advantage, as they can be deployed to characterize wheat-alien CILs bearing chromosomes from these genomes. Since this is the first report on the development of molecular markers from this genome, coupled with the efficiency of the markers as proven in our results, we recommend wide application of these markers in bread wheat breeding programs. Integrating the markers with in situ hybridization strategies would undoubtedly shorten the duration of cultivar development and produce more reliable results.

\section{Methods \\ Plant materials}

We analyzed 22 wheat- $L$. racemosus CILs (Table 7), three cultivated and nine wild Triticeae species (Table 8). The nine chromosome addition lines and 12 Triticeae species were obtained from the gene bank of Tottori Alien Chromosome Bank of Wheat (TACBOW), a subsidiary of the National BioResource Project (NBRP)KOMUGI, Japan, while the six translocation and seven recombination lines were personally provided by Dr. M. Kishii (co-author) of the International Maize and Wheat Improvement Center (CIMMYT). Apart from the two Leymus species and Elymus ciliaris, which are maintained permanently as live plants in Arid Land Research Center (ALRC), Tottori University, seeds of all other experimental plants were sown in trays filled with commercial soil (Takii and CAINZ, Japan), and the germinated plants were maintained until leaf samples

Table 7 Description and references of wheat-L. racemosus chromosome introgression lines (CILs)

\begin{tabular}{|c|c|c|c|c|}
\hline Genotype ID & Description & Alien chromosome ID & Chromosome constitution (2n) & Reference \\
\hline TACBOW 0001 & Disomic addition & Lr\#A & $21 "+1 "[\mathrm{~A}]$ & [9] \\
\hline TACBOW 0003 & Disomic addition & Lr\#E & $21 "+1 "[E]$ & {$[9]$} \\
\hline TACBOW 0004 & Disomic addition & Lr\#F & $21 "+1 "[F]$ & [9] \\
\hline TACBOW 0005 & Disomic addition & Lr\#H & $21 "+1 "[H]$ & {$[9]$} \\
\hline TACBOW 0006 & Disomic addition & Lr\#I & $21 "+1 "[1]$ & [9] \\
\hline TACBOW 0007 & Disomic addition & Lr\#」 & $21 "+1 "[J]$ & [9] \\
\hline TACBOW 0008 & Disomic addition & Lr\#K & $21 "+1 "[K]$ & {$[9]$} \\
\hline TACBOW 0009 & Disomic addition & Lr\#L & $21 "+1 "[L]$ & {$[9]$} \\
\hline TACBOW 0010 & Disomic addition & Lr\#N & $21 "+1 "[N]$ & [9] \\
\hline I short & I short arm translocation & Lr\#IS & 42 & {$[51]$} \\
\hline I long & I long arm translocation & Lr\#IL & 42 & {$[51]$} \\
\hline J short & J short arm translocation & Lr\#JS & 42 & {$[51]$} \\
\hline J long & $\mathrm{J}$ long arm translocation & Lr\#JL & 42 & {$[51]$} \\
\hline$N$ short & $\mathrm{N}$ short arm translocation & Lr\#NS & 42 & {$[51]$} \\
\hline N long & $\mathrm{N}$ long arm translocation & Lr\#NL & 42 & {$[51]$} \\
\hline N recomb \#1 & N recombination No. 1 & Lr\#NR1 & 42 & Current article \\
\hline $\mathrm{N}$ recomb \#2 & N recombination No. 2 & Lr\#NR2 & 42 & Current article \\
\hline $\mathrm{N}$ recomb \#3 & N recombination No. 3 & Lr\#NR3 & 42 & Current article \\
\hline $\mathrm{N}$ recomb \#4 & N recombination No. 4 & Lr\#NR4 & 42 & Current article \\
\hline $\mathrm{N}$ recomb \#5 & $\mathrm{N}$ recombination No. 5 & Lr\#NR5 & 42 & Current article \\
\hline $\mathrm{N}$ recomb \#6 & N recombination No. 6 & Lr\#NR6 & 42 & Current article \\
\hline N recomb \#7 & N recombination No. 7 & Lr\#NR7 & 42 & Current article \\
\hline
\end{tabular}

TACBOW Tottori Alien Chromosome Bank of Wheat (Japan), Lr Leymus racemosus, A, E, F, H, I, J, K, L, N Arbitrary numbering of L. racemosus chromosomes, IS I-short arm, IL I-long arm, JS J-short arm, JL J-short arm, NS N-short arm, NL N-long arm, NR N-recombination 
Table 8 Description and sources of 12 Triticeae species

\begin{tabular}{|c|c|c|c|c|}
\hline Species ID & Description & $2 n$ & Ploidy & Reference \\
\hline KT020-003 & Triticum aestivum cv. 'Chinese Spring' & 42 & $6 x$ & [18] \\
\hline TACBOW0071 & Secale cereale strain IR10 & 14 & $2 x$ & - \\
\hline TACBOW0112 & Leymus racemosus & 28 & $4 \times$ & [28] \\
\hline TACBOW0113 & L. mollis & 28 & $4 \times$ & [28] \\
\hline TACBOW0116 & Hordeum vulgare cv.'Betzes' & 14 & $2 x$ & [18] \\
\hline TACBOW0117 & H. bulbosum & 14 & $2 x$ & [18] \\
\hline TACBOW0119 & Dasypyrum villosum & 14 & $2 x$ & [18] \\
\hline TACBOW0121 & Psathyrostachys huashanica & 14 & $2 x$ & [10] \\
\hline TACBOW0122 & Elymus ciliaris & 28 & $4 \times$ & [18] \\
\hline KU-199-1 & T. urartu & 14 & $2 x$ & - \\
\hline $\mathrm{KU}-2-1$ & Aegilops speltoides & 14 & $2 x$ & - \\
\hline KU-20-2 & Ae. Tauschii & 14 & $2 x$ & - \\
\hline
\end{tabular}

TACBOW Tottori Alien Chromosome Bank of Wheat (Japan). All the strains and their detailed description and origin are available in the gene bank of National BioResource Project (NBRP-KOMUGI) (https://shigen.nig.ac.jp/wheat/komugi/)

were ready for DNA extraction. About two weeks after sowing, leaf samples were collected from each plant, immediately frozen in liquid nitrogen and stored at $-80{ }^{\circ} \mathrm{C}$ until needed for DNA extraction. Cetyl trimethylammonium bromide (CTAB) miniprep extraction protocol, with some modifications, was followed to extract and purify genomic DNA from all samples, while quantification and quality check were done with NanoDrop2000C Spectrophotometer (ThermoScientific, USA).

\section{Production of wheat-L. racemosus chromosome introgression lines}

Details of the production procedures and identification of $L$. racemosus chromosomes in the chromosome addition and translocation lines were reported in previous studies [9, 28, 51]. We, therefore, report here the additional steps taken to develop the N-recombination lines, which we characterized alongside with the addition and translocation lines in this study. Basically, our strategy was modelled after the methodology described and adopted in the production of wheat-rye recombination lines [24]. To produce the first $\mathrm{N}$-recombination line, $\mathrm{N}$ recomb \#1, we crossed a monosomic Chinese Spring (CS) wheat line $\left(2 \mathrm{n}=42-3 \mathrm{~B}^{\prime}\right)$ to a disomic N-addition line $\left(2 \mathrm{n}=42+\mathrm{N}^{\prime \prime}\right)$ and selected $\mathrm{N}$ monosomic substitution plants $\left(2 n=42+N^{\prime}-3 B^{\prime}\right)$ in the first filial generation $\left(F_{1}\right)$. In the second filial generation $\left(F_{2}\right)$, a naturally occurring recombinant was recognized by $\mathrm{FISH} / \mathrm{GISH}$ analysis and homozygote recombinants were selected and named $\mathrm{N}$-recomb \#1. The production of $\mathrm{N}$-recomb $\# 2$ to \#7, except \#4, was initiated by the hybridization of $\mathrm{N}$-short arm translocation with a CS ph 1 mutant (CSph 1) to enable homoeologous pairing and recombination. The $F_{1}$ plants were then self-pollinated and five sets of recombinant homozygotes, with different recombination rates, were selected. For N-recomb \#4, we crossed another bread wheat cultivar, WEBILL1 (a dominant bread wheat line at CIMMYT in the 2000's), with a disomic N-addition and selected monosomic addition plants at the $\mathrm{F}_{1}$, which were then backcrossed to WEBILL1 to generate $\mathrm{BC}_{1} \mathrm{~F}_{1}$ monosomic addition plants. The $\mathrm{BC}_{1} \mathrm{~F}_{1}$ monosomic plants were then hybridized with CS-ph 1 mutant and N-recomb \#4 homozygote plants were selected at the $F_{2}$, following the steps described for the last five genotypes.

\section{Growth conditions for Leymus racemosus seedlings used for sequencing}

Seedlings of Leymus racemosus were raised in a hydroponic culture $\left(2 \mathrm{mM} \mathrm{KNO}, 0.28 \mathrm{mMKH}_{2} \mathrm{PO}_{4}, 0.18 \mathrm{mM}\right.$ $\mathrm{K}_{2} \mathrm{SO}_{4}, 0.07 \mathrm{mM} \mathrm{CaCl} 2 \quad 2 \mathrm{H}_{2} \mathrm{O}, 0.15 \mathrm{mM} \mathrm{MgSO}_{4}$ $7 \mathrm{H}_{2} \mathrm{O}, 75 \mathrm{nM} \mathrm{H} \mathrm{BO}_{3}, 12.5 \mathrm{nM} \mathrm{MnSO}{ }_{4} 6 \mathrm{H}_{2} \mathrm{O}, 25 \mathrm{nM}$ $\mathrm{Na}_{2} \mathrm{MoO}_{4} \quad 2 \mathrm{H}_{2} \mathrm{O}, 10 \mathrm{nM} \mathrm{ZnSO}_{4} \quad 7 \mathrm{H}_{2} \mathrm{O}, 0.5 \mathrm{mM}$ FeEDTA, $\left.5 \mathrm{nM} \quad \mathrm{CuSO}_{4} \quad 5 \mathrm{H}_{2} \mathrm{O}\right)$, with a day/night temperature regime of $25 / 15{ }^{\circ} \mathrm{C}(14 \mathrm{~h}$ light/10 h dark photoperiod) in Arid Land Research Center growth chamber for two months. Hydroponic solution was changed every week. To obtain young root tissues, oldlignified roots were partially cut and plants were grown in the hydroponic culture for two weeks. Young roots were then separately subjected to salinity stress and ammonium treatment for $12 \mathrm{~h}$, to ensure gene expression for salinity tolerance and BNI activity, which are reported traits of L. racemosus [17, 42]. Salinity stress was imposed by addition of $400 \mathrm{mM} \mathrm{NaCl}$ to the hydroponic medium, while ammonium treatment was achieved by replacement of $2 \mathrm{mM} \mathrm{KNO}_{3}$ with $2 \mathrm{mM}\left(\mathrm{NH}_{4}\right)_{2} \mathrm{SO}_{4}$. Control plants were maintained in unaltered hydroponic medium. Root tissues were harvested, frozen in liquid nitrogen and stored at $-80^{\circ} \mathrm{Cuntil}$ needed for DNA and RNA extraction. 


\section{RNA extraction and library preparation}

Control-, salt- and ammonium-treated root tissues of $L$. racemosus were used for RNA-sequencing. Total RNA was extracted using RNeasy mini kit with the inclusion of an on-column DNase digestion kit (Qiagen). Using the isolated RNA, mRNA-seq libraries were constructed for the three conditions using TruSeq RNA Sample Preparation. To generate 150-bp pair-end reads, the libraries were sequenced by HiSeq2500 according to the standard protocol.

\section{Assembly of RNA-sequencing reads}

A total of 174-GB reads were determined by mRNAsequencing (Additional file 12). Approximately 5\% of reads with low-quality scores or adapters were partially trimmed by Trimmomatic software (version 0.32) [52]. To remove non-mRNA sequences, we collected known rRNA and tRNA sequences [53, 54], and after removing the reads mapped to rRNA and tRNA sequences by Bowtie2 software (version 2.2.3) [55], the remaining reads were used for construction of assembled contigs in either control, high ammonium or salinity stress condition. First, assembled contigs were generated by three softwares: Velvet ver. 1.2.10-Oases ver. 0.2.8., SOAPdenovo-Trans Ver. 1.0.3 and Trinity ver. 2.1.1 [56] in sequencing reads derived from control condition. Contigs assembled by Velvet-Oases used various kmersizes (39, 49, 59, 69, 79, 89 and 99)(Velvet version 1.2.10, Oases version 0.2 .8 ), and all the contigs assembled by various kmer-sizes were merged into a contig. Contigs assembled by either SOAPdenovo-Trans or Trinity used optimized K-mers. There are three assembled contigs in Velvet-Oases, SOAPdenovo-Trans and Trinity. To identify the best contig among three contigs, we examined the conserved regions of $L$. racemosus against $T$. aestivum cDNA sequences (version 30, Ensembl) by BLASTN software [57]. The proportions of conserved sequences were $44 \%, 17 \%$ and $4 \%$ in Velvet-Oases, SOAPdenovo-Trans and Trinity contigs, respectively. Here, Velvet-Oases generated the best contigs with respect to similarity in closely related species. In the same procedure, we generated $634,480,460,748$ and 434,862 contigs with more than 500 bp in normal, high ammonium and salinity stress condition, respectively.

\section{Primer design from RNA-seq}

We designed primers in the homologous regions between $T$. aestivum and $L$. racemosus and $L$. racemosus specific region, resulting in two categories of primers. First, both forward and reverse primers are in the homologous regions but the length of amplified DNA fragment is expected to be different between $T$. aestivum and $L$. racemosus by $20-1000 \mathrm{bp}$. Second, either forward or reverse primer is in the homologous region, while the other primer is in L. racemosus specific sequence, and the amplified DNA fragment is expected to range from 100 to $1000 \mathrm{bp}$. All the primers were designed by Primer3 software [58]. We designed 9256 and 7637 pairs of primers in the contigs with more than $500 \mathrm{bp}$ in normal condition in the former and latter strategies, respectively. To design primers in high salinity and ammonium contigs, we first removed high salinity contigs which are similar to normal condition contigs and then removed high ammonium contigs which are similar to either normal or high salinity contigs. From the remaining high salinity contigs, using the two strategies in the order explained above, we designed 4930 and 3339 pairs of primers, respectively, and from the filtered high ammonium contigs, we designed 5461 and 4312 pairs of primers, respectively. Thus, we identified a total of 34,935 pairs of primers to identify the difference between $T$. aestivum and $L$. racemosus.

\section{Genome sequencing and assembly}

Genomic DNA was extracted from root tissues of 2week-old $L$. racemosus plants (control treatment) using MagExtractor $^{\mathrm{Tn}}$-Plant Genome- (TOYOBO). The isolated DNA was submitted to generate sequencing library for Illumina MiSeq analysis, and the library construction and sequencing process were achieved by a purchasable service from Macrogen, Japan. A total of $\sim 35 \mathrm{M}$ pairedend reads $(2 \times 151-\mathrm{nt})$ was obtained from the analysis. Subsequent quality trimming $(\mathrm{Q}>30)$ and artificial sequence elimination steps were achieved manually. The cleaned reads were subjected to build L. racemosus genome contigs utilizing Platanus software (v1.2.4) [59]. Assemblies with variable k-mers $(27,29,31,33,35$, and 37$)$ were conducted in parallel, and the resultants were merged into $L$. racemosus genome scaffolds of unique and significantly long (>1000-nt) length. The raw sequence data was deposited in NCBI/EBI/DDBJ short read archive under a specific accession number (SRR5796629).

\section{Primer design from genomic sequence}

Wheat mRNA-seq data from a previous study [60] was mapped to the $L$. racemosus genome scaffolds with the aid of TopHat (ver. 2.0.8) [61] with the following options: "-read-realign-edit-dist 0 -b2-fast -meta-std-dev 200 -a 6 -i 8 -I 10000 -max-segment-intron 100 -min-segmentintron 3". Ten scaffolds were retained based on their length (> 2000-nt) and read mapping (no specific mRNAseq read mapping). Scaffolds polymorphisms against wheat reference genome (v1.1) [62] were evaluated by BLASTN, and primers sensitive to relatively large (>3-nt) gaps were designed by Primer3. 
Development of markers and genotyping of wheat- $L$. racemosus chromosome introgression lines by $P C R$

Utilizing genomic DNA samples from bread wheat and L. racemosus, we screened 294 randomly selected primer sets - 150 from DNA sequence and 144 from RNA-seq. Each $20 \mu \mathrm{L}$ reaction volume contained $10 \mu \mathrm{L}$ KAPA Taq Extra HotStart ReadyMix with dye (KapaBiosystems), 1 $\mu \mathrm{L}(10 \mu \mathrm{M})$ each of forward and reverse primers, $2 \mu \mathrm{L}$ (50 ng) DNA template and $6 \mu \mathrm{L}$ PCR grade water. With BIORAD T100 Thermal Cycler, the samples, in a 96well plate, were subjected to touchdown PCR: $95{ }^{\circ} \mathrm{C}$ initialization for $3 \mathrm{~min}, 5$ cycles of $95^{\circ} \mathrm{C}$ denaturation for $30 \mathrm{~s}, 65{ }^{\circ} \mathrm{C}$ to $61{ }^{\circ} \mathrm{C}\left(-1{ }^{\circ} \mathrm{C} /\right.$ cycle $)$ annealing for $30 \mathrm{~s}$ and $72{ }^{\circ} \mathrm{C}$ extension for $30 \mathrm{~s}$; 30 cycles of $95{ }^{\circ} \mathrm{C}$ denaturation (30 s), $60{ }^{\circ} \mathrm{C}$ annealing (30 s) and $72{ }^{\circ} \mathrm{C}$ extension (30 s) and final extension at $72{ }^{\circ} \mathrm{C}$ for $10 \mathrm{~s}$. Because the average melting temperature $(\mathrm{Tm})$ of primers designed from DNA sequence was about $8{ }^{\circ} \mathrm{C}$ higher than the average Tm of primers designed from RNA-seq, we substituted the annealing range of $65-60{ }^{\circ} \mathrm{C}$ in the cycling program with $57-52{ }^{\circ} \mathrm{C}$ for the RNA-seq primers. All PCR products were electrophoresed for $30 \mathrm{~min}$ on $1.5 \%$ agarose $\mathrm{S}$ gel in Tris-acetate-EDTA (1X TAE) buffer, stained in ethidium bromide solution for $10 \mathrm{~min}$ and photographed with AE-6932GXCF transilluminator.

From the pre-screened primers, polymorphic markers were selected and applied to genotype nine wheat- $L$. racemosus chromosome addition lines and markers specific to L. racemosus chromosomes I, J and $\mathrm{N}$ were deployed to characterize two each of $\mathrm{I}-, \mathrm{J}-$ and $\mathrm{N}$ translocation and seven N-recombination lines, respectively. To assess the transferability of $L$. racemosus markers to other Triticeae species, we used 164 markers amplified in $L$. racemosus to genotype 12 species in the tribe (Table 8 ), including $L$. racemosus as a positive control, aiming at analyzing polymorphism between bread wheat genome and genomes of other species studied.

\section{Sequencing and analysis of some PCR products}

We applied Sanger sequencing to determine the nucleotide sequence of PCR products generated by one of our markers which amplified all the L. racemosus chromosomes added to wheat. All the PCR products were purified with AxyPrep PCR cleanup kit, according to the PCR cleanup spin protocol (AXYGEN Biosciences). The purified products were premixed in accordance with Macrogen's recommendation (Macrogen, Japan) and same delivered to the company for sequencing. Each genotype sequence was searched against nucleotide sequences in NCBI and Ensembl Plants databases using BLASTN (megablast). Also, the DNA scaffold from which the marker was developed was searched in like manner. To check for polymorphism between the chromosomes, we aligned all the sequences using JustBio multiple alignment tool.

\section{Development of markers and genotyping of wheat- $L$. racemosus chromosome introgression lines by DArT-seq} To complement the PCR-based markers and widen the scope of application of our makers, especially chromosome-specific markers, we applied DArT-seq to genotype L. racemosus and the 22 CILs alongside bread wheat to assist in data analysis and interpretation. DArT-seq platform used HiSeq2500 to sequence the samples and generated 44,277 markers. This approach enabled us to develop massive chromosome-specific markers for the nine $L$. racemosus chromosomes analyzed.

\section{Analysis of data \\ PCR data}

PCR results were scored in a binary fashion, " 0 " and " 1 " for absence and presence of band, respectively, while size polymorphic bands (very few) were differentiated using 1 to designate band size in wheat and 2 for band size in an introgression line or another Triticeae species, depending on the case. The scores were analyzed using simple proportion to determine the percentage of screened primers amplified in $L$. racemosus genome as well as the proportion of the amplified markers polymorphic in wheat. Also, the frequency of amplification of alien chromosomes in the CILs was computed, with a view to making clear the proportion of the developed markers located on the alien chromosomes. Markers specifically amplified by each of the nine chromosome addition lines were designated chromosome-specific and I-, J- and N-specific markers specifically located on any of the translocation lines were accordingly named armspecific. Also, arm-specific markers of chromosome $\mathrm{N}$ specifically amplified by the seven $\mathrm{N}$-recombination lines were applied to determine the arm location of each recombinant fragment. Data from the screening of the 12 Triticeae species were handled in a similar manner, but with more emphasis in identifying and computing polymorphism in wheat in each case. This gave a basis to decide the suitability of $L$. racemosus for genotyping of wheat lines carrying chromosomes from these species. In addition, we used PCR-based markers amplified in each species to compare frequency of polymorphism between DNA and RNA sequence information to assess the suitability of the two approaches.

\section{DArT-seq data}

DArT-seq markers in the SNP 1-Row Mapping Format, which we used for our analysis, were scored " 0 ", " 1 " and "2", representing reference (Wheat_ChineseSpring04) allele only, SNP allele only and both reference and SNP 
alleles, respectively. This simply means that any genomic representation scored " 0 " for a marker lacks the alien chromosome identified by the corresponding SNP allele, while those scored " 1 " or " 2 " have the SNP or both the SNP and reference alleles, respectively. We set the call rate at greater than or equal to $85 \%$ to aid us filter reliable markers from the stream of the entire data obtained from DArT-seq platform. Markers with no clear information regarding their physical positions on corresponding wheat chromosomes, no polymorphism in wheat genome and those without SNP alleles in L. racemosus were filtered against. In order to obtain the fraction of the polymorphic markers that provided useful genotypic information on the CILs, we sieved all the markers located on genomic representations of the lines, carefully separating chromosome-specific markers from coamplified. We also analyzed homology between I, J, N and their respective translocation lines, aiming at identifying markers specific to each of the arms as done with the PCR markers and determining the locations of the $\mathrm{N}$-recombinant fragments to allow comparison with the PCR-based result. With the corresponding physical positions of the N-specific markers revealed by DArT-seq data, we graphically genotyped the $\mathrm{N}$-recombination lines. In the final analysis, correspondence of all the chromosome-specific markers with the homoeologous groups of CS chromosomes was utilized to determine the most probable homoelogous groups of L. racemosus chromosomes in the CILs.

\section{Additional files}

Additional file 1: Table S1. Detailed information of Lr\#A-specific markers. (XLSX $44 \mathrm{~kb}$ )

Additional file 2: Table S2. Detailed information of Lr\#E-specific markers. (XLSX $10 \mathrm{~kb}$ )

Additional file 3: Table S3. Detailed information of Lr\#F-specific markers. (XLSX $39 \mathrm{~kb}$ )

Additional file 4: Table S4. Detailed information of $\mathrm{LrHH}$-specific markers. (XLSX $40 \mathrm{~kb}$ )

Additional file 5: Table S5. Detailed information of Lr\#l-specific markers. (XLSX $49 \mathrm{~kb}$ )

Additional file 6: Table S6. Detailed information of Lr\#J-specific markers. (XLSX $44 \mathrm{~kb}$ )

Additional file 7: Table S7. Detailed information of Lr\#K-specific markers. (XLSX $46 \mathrm{~kb}$ )

Additional file 8: Table S8. Detailed information of Lr\#L-specific markers. (XLSX $52 \mathrm{~kb}$ )

Additional file 9: Table S9. Detailed information of $\mathrm{Lr \# N-specific}$ markers. (XLSX $44 \mathrm{~kb}$ )

Additional file 10: Fig. S1. GISH photos showing molecular cytogenetic identification of alien segments in N-recombination lines. (PPTX $7852 \mathrm{~kb}$ )

Additional file 11: Table S10. Sequences of Leymus racemosus chromosomes' universal markers. (DOCX $14 \mathrm{~kb}$ )

Additional file 12: Table S11. Raw mRNA sequence reads of $L$. racemosus. (DOCX $15 \mathrm{~kb}$ )

\section{Abbreviations}

CTAB: Cetyl trimethylammonium bromide; DArT-seq: Diversity arrays technology-sequence based; DNA: Deoxyribonucleic acid; EDTA: Ethylene diaminetetraacetic acid; PCR: Polymerase chain reaction; RNA: Ribonucleic acid; TACBOW: Tottori alien chromosome bank of wheat

\section{Acknowledgements}

This study was partly funded by JSPS KAKENHI (No. 25292039), Joint Research Program of ALRC, Tottori University (Nos. 29D2001 and 28C2007). We also thank NODAI Gnome Science Center and National Institute of Genetics for Illumina sequencing service and supercomputer services, respectively.

\section{Funding}

This study was partly funded by JSPS KAKENHI Grant Number 25292039. The funding agency assessed our proposal and based on merit, approved and funded the study.

\section{Availability of data and materials}

The datasets supporting our conclusions are included within the article and its additional files and plant materials can be sourced from the references stated in the article.

\section{Authors' contributions}

OUE, JSK, MO, YSAG and HT conceived and designed the experiments. JSK and $M O$ raised $L$. racemosus plants for genomic and RNA sequencing and analyzed genomic sequence, while $\mathrm{KH}$ and $\mathrm{TT}$ prepared RNA libraries, analyzed RNA-seq and designed primers therefrom. MK produced and conducted cytogenetic analysis of $\mathrm{N}$-recombination lines. OUE designed primers from genomic sequence, screened primers, developed markers from DNA and RNA-seq and genotyped the plant materials by PCR and DArT-seq. OUE, JSK, MO, KH, YSAG and HT jointly wrote the manuscript. All the authors read and approved the final manuscript.

\section{Ethics approval and consent to participate}

Not applicable: This study does not directly involve humans or animals. All the plant materials utilized are maintained in the gene bank of Tottori Alien Chromosome Bank of Wheat (TACBOW), Arid Land Research Center, Tottori University, managed by the corresponding author of this article. They are also replicated in the gene bank of National BioResource Project (NBRPKOMUGI) (https://shigen.nig.ac.jp/wheat/komugi/), Japan. As stated under the methods section of this article, we obtained all the plant materials from TACBOW gene bank, through Prof. H. Tsujimoto (corresponding author) and Dr. M. Kishii (co-author); hence, we had no need for field collection. Alternative sources of information for some of the materials are also provided as references cited against them in Tables 7 and 8.

Consent for publication

Not applicable.

\section{Competing interests}

The authors declare that they have no competing interests.

\section{Publisher's Note}

Springer Nature remains neutral with regard to jurisdictional claims in published maps and institutional affiliations.

\section{Author details}

${ }^{1}$ Arid Land Research Center, Tottori University, Tottori, Japan. ${ }^{2}$ RIKEN Center for Sustainable Resource Science, Tsukuba, Ibaraki 305-0074, Japan. ${ }^{3}$ Center for Bioscience Research and Education, Utsunomiya University, Utsunomiya, Japan. ${ }^{4}$ Department of Bioscience and Bioinformatics, Kyushu Institute of Technology, Kitakyushu, Japan. ${ }^{5}$ International Maize and Wheat Improvement Center (CIMMYT), El Batan, Mexico. ${ }^{6}$ Agricultural Research Corporation (ARC), Wad Madani, Sudan. ${ }^{7}$ United Graduate School of Agricultural Sciences, Tottori University, Tottori, Japan. 


\section{Received: 21 November 2017 Accepted: 13 March 2018} Published online: 27 March 2018

\section{References}

1. Ali N, Heslop-Harrison JS, Ahmad H, Graybosch RA, Hein GL, Schwarzacher T. Introgression of chromosome segments from multiple alien species in wheat breeding lines with wheat streak mosaic virus resistance. Heredity. 2016;117(2):114-23.

2. King J, Armstead I, Harper J, Ramsey L, Snape J, Waugh R, James C, Thomas A, Gasior D, Kelly R, et al. Exploitation of interspecific diversity for monocot crop improvement. Heredity (Edinb). 2013;110(5):475-83.

3. Hajjar R, Hodgkin T. The use of wild relatives in crop improvement: a survey of developments over the last 20 years. Euphytica. 2007;156(1-2):1-13.

4. Niu Z, Klindworth DL, Friesen TL, Chao S, Jin Y, Cai X, Xu SS. Targeted introgression of a wheat stem rust resistance gene by DNA marker-assisted chromosome engineering. Genetics. 2011;187(4):1011-21.

5. Olson EL, Rouse MN, Pumphrey MO, Bowden RL, Gill BS, Poland JA. Introgression of stem rust resistance genes SrTA10187 and SrTA10171 from Aegilops tauschii to wheat. Theor Appl Genet. 2013;126(10):2477-84

6. Pradhan GP, Prasad PV. Evaluation of wheat chromosome translocation lines for high temperature stress tolerance at grain filling stage. PLoS One. 2015; 10(2):e0116620.

7. Rahmatov M, Rouse MN, Steffenson BJ, Andersson SC, Wanyera R, Pretorius ZA, Houben A, Kumarse N, Bhavani S, Johansson E. Sources of stem rust resistance in wheat-alien introgression lines. Plant Dis. 2016;100(6):1101-9.

8. Zhang $H$, Mittal N, Leamy LJ, Barazani O, Song BH. Back into the wild-apply untapped genetic diversity of wild relatives for crop improvement. Evol Appl. 2017;10(1):5-24.

9. Kishii M, Yamada T, Sasakuma T, Tsujimoto H. Production of wheat-Leymus racemosus chromosome addition lines. Theor Appl Genet. 2004;109(2):25560

10. Kishii M, Dou Q, Garg M, Ito M, Tanaka H, Tsujimoto H. Production of wheatPsathyrostachys huashanica chromosome addition lines. Genes Genet Syst. 2010;85(4):281-6.

11. Zhang P, Dundas IS, Xu SS, Friebe B, RA MI, Raupp WJ. chromosome engineering techniques for targeted introgression of rust resistance from wild wheat relatives. Methods Mol Biol. 2017;1659:163-72.

12. Larson SR, Kishii M, Tsujimoto H, Qi L, Chen P, Lazo GR, Jensen KB, Wang RR. Leymus EST linkage maps identify 4NsL-5NsL reciprocal translocation, wheat-Leymus chromosome introgressions, and functionally important gene loci. Theor Appl Genet. 2012;124(1):189-206.

13. Anamthawat-Jonsson K. Molecular cytogenetics of Leymus: mapping the ns genome-specific repetitive sequences. J Syst Evol. 2014;52(6):716-21.

14. Anamthawat-Jonsson K, Bodvarsdottir SK. Genomic and genetic relationships among species of Leymus (Poaceae: Triticeae) inferred from 18S-26S ribosomal genes. Am J Bot. 2001;88(4):553-9.

15. Chen P, Liu W, Yuan J, Wang X, Zhou B, Wang S, Zhang S, Feng Y, Yang B, Liu G, et al. Development and characterization of wheat- Leymus racemosus translocation lines with resistance to fusarium head blight. Theor Appl Genet. 2005:111(5):941-8

16. Qi LL, Pumphrey MO, Friebe B, Chen PD, Gill BS. Molecular cytogenetic characterization of alien introgressions with gene Fhb3 for resistance to fusarium head blight disease of wheat. Theor Appl Genet. 2008;117(7): 1155-66.

17. McGuire P, Dvorak J. high salt-tolerance potential in wheatgrasses. Crop Sci. 1981:21:702-5.

18. Hagras AAA, Kishii M, Sato K, Tanaka H, Tsujimoto H. Extended application of barley EST markers for the analysis of alien chromosomes added to wheat genetic background. Breeding Sci. 2005;55(3):335-41.

19. Wang RR, Larson SR, Jensen KB. Differential transferability of EST-SSR primers developed from the diploid species Pseudoroegneria spicata, Thinopyrum bessarabicum, and Thinopyrum elongatum. Genome. 2017:60(6):530-6.

20. Pang YH, Zhao JX, Du WL, Li YL, Wang J, Wang LM, Wu J, Cheng XN, Yang $\mathrm{QH}$, Chen $\mathrm{XH}$. Cytogenetic and molecular identification of a wheat-Leymus mollis alien multiple substitution line from octoploid Tritileymus $x$ Triticum durum. Genet Mol Res. 2014;13(2):3903-13.

21. Bushman BS, Larson SR, Mott IW, Cliften PF, Wang RR, Chatterton NJ Hernandez AG, Ali S, Kim RW, Thimmapuram J, et al. Development and annotation of perennial Triticeae ESTs and SSR markers. Genome. 2008; 51(10):779-88
22. Rey MD, Prieto $P$. Detection of alien genetic introgressions in bread wheat using dot-blot genomic hybridisation. Mol Breed. 2017;37(3):32

23. Yang $C$, Zhang $H$, Chen $W$, Kang $H$, Wang $Y$, Sha L, Fan $X$, Zeng J, Zhou $Y$. Genomic constitution and intergenomic translocations in the Elymus dahuricus complex revealed by multicolor GISH. Genome. 2017;60(6):510-7.

24. Lukaszewski A. Manipulation of the 1RS.1BL translocation in wheat by induced homoeologous recombination. Crop Sci. 1999;40(1):216-25.

25. Anamthawat-Jonsson K. Genetic and genomic relationships in Leymus Hochst. Hereditas. 2001;135(2-3):247-53.

26. Alix K, Gerard PR, Schwarzacher T, Heslop-Harrison JS. Polyploidy and interspecific hybridization: partners for adaptation, speciation and evolution in plants. Ann Bot. 2017;120:184-94.

27. Shiferaw B, Smale M, Braun HJ, Duveiller E, Reynolds M, Muricho G. Crops that feed the world 10. Past successes and future challenges to the role played by wheat in global food security. Food Secur. 2013;5(3):291-317.

28. Kishii M, Nagaki K, Tsujimoto H, Sasakuma T. Exclusive localization of tandem repetitive sequences in subtelomeric heterochromatin regions of Leymus racemosus (Poaceae, Triticeae). Chromosom Res. 1999;7(7):519-29.

29. Lukaszewski AJ, Lapinski B, Rybka K. Limitations of in situ hybridization with total genomic DNA in routine screening for alien introgressions in wheat. Cytogenet Genome Res. 2005;109(1-3):373-7.

30. Zhang P, Li W, Friebe B, Gill BS. The origin of a "zebra" chromosome in wheat suggests nonhomologous recombination as a novel mechanism for new chromosome evolution and step changes in chromosome number. Genetics. 2008;179(3):1169-77.

31. Zhang L, Liu D, Yan Z, Zheng Y. Alien DNA introgression and wheat DNA rearrangements in a stable wheat line derived from the early generation of distant hybridization. Sci China C Life Sci. 2005;48(5):424-33.

32. Garg M, Elamein HM, Tanaka H, Tsujimoto H. Preferential elimination of chromosome 1D from homoeologous group-1 alien addition lines in hexaploid wheat. Genes Genet Syst. 2007;82(5):403-8.

33. Gorafi YS, Eltayeb AE, Tsujimoto $H$. Alteration of wheat vernalization requirement by alien chromosome-mediated transposition of MITE. Breed Sci. 2016:66(2):181-90.

34. Gernand D, Rutten T, Varshney A, Rubtsova M, Prodanovic S, Bruss C, Kumlehn J, Matzk F, Houben A. Uniparental chromosome elimination at mitosis and interphase in wheat and pearl millet crosses involves micronucleus formation, progressive heterochromatinization, and DNA fragmentation. Plant Cell. 2005;17(9):2431-8.

35. Ishii T, Ueda T, Tanaka H, Tsujimoto H. Chromosome elimination by wide hybridization between Triticeae or oat plant and pearl millet: pearl millet chromosome dynamics in hybrid embryo cells. Chromosom Res. 2010;18(7): 821-31.

36. Sanei M, Pickering R, Kumke K, Nasuda S, Houben A. Loss of centromeric histone $\mathrm{H} 3$ (CENH3) from centromeres precedes uniparental chromosome elimination in interspecific barley hybrids. Proc Natl Acad Sci U S A. 2011; 108(33):E498-505.

37. Wicker T, Guyot R, Yahiaoui N, Keller B. CACTA transposons in Triticeae. A diverse family of high-copy repetitive elements. Plant Physiol. 2003;132(1):52-63.

38. Fedoroff NV. Molecular genetics and epigenetics of CACTA elements. Methods Mol Biol. 2013;1057:177-92.

39. Sergeeva EM, Salina EA, Adonina IG, Chalhoub B. Evolutionary analysis of the CACTA DNA-transposon Caspar across wheat species using sequence comparison and in situ hybridization. Mol Gen Genomics. 2010;284(1):11-23.

40. Langdon T, Jenkins G, Hasterok R, Jones RN, King IP. A high-copy-number CACTA family transposon in temperate grasses and cereals. Genetics. 2003; 163(3):1097-108.

41. Miura A, Kato M, Watanabe K, Kawabe A, Kotani H, Kakutani T. Genomic localization of endogenous mobile CACTA family transposons in natural variants of Arabidopsis thaliana. Mol Gen Genomics. 2004:270(6):524-32.

42. Subbarao GV, Ban T, Kishii M, Ito O, Samejima H, Wang HY, Pearse SJ, Gopalakrishnan S, Nakahara K, Tsujimoto H, Berry W. Can biological nitrification inhibition (BNI) genes from perennial Leymus racemosus (Triticeae) combat nitrification in wheat farming? Plant Soil. 2007:299:55-64.

43. Subbarao GV, Rondon M, Ito O, Ishikawa T, Rao IM, Nakahara K, Lascano C, Berry WL. Biological nitrification inhibition (BNI) - is it a widespred phenomenon? Plant Soil. 2007;294:15-9.

44. Almeida NF, Trindade Leitao S, Caminero C, Torres AM, Rubiales D, Vaz Patto MC. Transferability of molecular markers from major legumes to Lathyrus spp. for their application in mapping and diversity studies. Mol Biol Rep. 2014:41(1):269-83. 
45. Xiao Y, Xia W, Ma J, Mason AS, Fan H, Shi P, Lei X, Ma Z, Peng M. Genomewide identification and transferability of microsatellite markers between Palmae species. Front Plant Sci. 2016;7:1578.

46. Zeng D, Luo J, Li Z, Chen G, Zhang L, Ning S, Yuan Z, Zheng Y, Hao M, Liu D. High transferability of Homoeolog-specific markers between bread wheat and newly synthesized Hexaploid wheat lines. PLoS One. 2016;11(9): e0162847.

47. da Silva RA, Souza G, Lemos LS, Lopes UV, Patrocinio NG, Alves RM, Marcellino LH, Clement D, Micheli F, Gramacho KP. Genome size, cytogenetic data and transferability of EST-SSRs markers in wild and cultivated species of the genus Theobroma L. (Byttnerioideae, Malvaceae). PLoS One. 2017;12(2):e0170799.

48. Baum BR, Edwards T, Johnson DA. Diversity within the genus Elymus (Poaceae: Triticeae) II: analyses of variation within $5 \mathrm{~S}$ nrDNA restrict membership in the genus to species with StH genomes. Mol Gen Genomics. 2016;291(1):217-25.

49. Wu X, Larson SR, Hu Z, Palazzo AJ, Jones TA, Wang RR, Jensen KB, Chatterton NJ. Molecular genetic linkage maps for allotetraploid Leymus wildryes (Gramineae: Triticeae). Genome. 2003;46(4):627-46.

50. Wang RR, Zhang JY, Lee BS, Jensen KB, Kishii M, Tsujimoto H. Variations in abundance of 2 repetitive sequences in Leymus and Psathyrostachys species. Genome. 2006:49(5):511-9.

51. Kishii M. Production of wheat-Leymus racemosus translocation lines. In: Electronic Wheat Information Service, vol. Vol. 111: eWIS; 2011. p. 11-3.

52. Bolger AM, Lohse M, Usadel B. Trimmomatic: a flexible trimmer for Illumina sequence data. Bioinformatics. 2014;30(15):2114-20.

53. Ludwig W, Strunk O, Westram R, Richter L, Meier H, Yadhukumar, Buchner A, Lai T, Steppi S, Jobb G, et al. ARB: a software environment for sequence data. Nucleic Acids Res. 2004;32(4):1363-71.

54. Chan PP, Lowe TM. GtRNAdb: a database of transfer RNA genes detected in genomic sequence. Nucleic Acids Res. 2009;37(Database issue):D93-7.

55. Langmead B, Salzberg SL. Fast gapped-read alignment with bowtie 2. Nat Methods. 2012;9(4):357-9.

56. Schulz MH, Zerbino DR, Vingron M, Birney E. Oases: robust de novo RNAseq assembly across the dynamic range of expression levels. Bioinformatics. 2012;28(8):1086-92.

57. Altschul SF, Madden TL, Schaffer AA, Zhang J, Zhang Z, Miller W, Lipman DJ. Gapped BLAST and PSI-BLAST: a new generation of protein database search programs. Nucleic Acids Res. 1997;25(17):3389-402.

58. Untergasser A, Cutcutache I, Koressaar T, Ye J, Faircloth BC, Remm M, Rozen SG. Primer3-new capabilities and interfaces. Nucleic Acids Res. 2012;40(15): e115.

59. Kajitani R, Toshimoto K, Noguchi H, Toyoda A, Ogura Y, Okuno M, Yabana M, Harada M, Nagayasu E, Maruyama $H$, et al. Efficient de novo assembly of highly heterozygous genomes from whole-genome shotgun short reads. Genome Res. 2014;24(8):1384-95.

60. Oono Y, Kobayashi F, Kawahara Y, Yazawa T, Handa H, Itoh T, Matsumoto T. Characterisation of the wheat (Triticum aestivum L.) transcriptome by de novo assembly for the discovery of phosphate starvation-responsive genes: gene expression in pi-stressed wheat. BMC Genomics. 2013;14:77.

61. Trapnell C, Pachter L, Salzberg SL. TopHat: discovering splice junctions with RNA-Seq. Bioinformatics. 2009;25(9):1105-11.

62. International Wheat Genome Sequencing C. a chromosome-based draft sequence of the hexaploid bread wheat (Triticum aestivum) genome. Science. 2014;345(6194):1251788.

\section{Submit your next manuscript to BioMed Central and we will help you at every step:}

- We accept pre-submission inquiries

- Our selector tool helps you to find the most relevant journal

- We provide round the clock customer support

- Convenient online submission

- Thorough peer review

- Inclusion in PubMed and all major indexing services

- Maximum visibility for your research

Submit your manuscript at www.biomedcentral.com/submit 